\title{
Magnetic properties of $\mathrm{Tm}_{2} \mathrm{Fe}_{16}$ under pressure
}

\author{
Anatoly Kuchin ${ }^{1, *}$, Zdenek Arnold $^{2}$, Jiri Kamarád², Sergey Platonov ${ }^{1}$ \\ ${ }^{1}$ M.N. Miheev Institute of Metal Physics UD RAS, Ekaterinburg, Russia \\ ${ }^{2}$ Institute of Physics AS CR v.v.i., Prague 8, Czech Republic
}

\begin{abstract}
The magnetic properties of the non-stoichiometric $\mathrm{Tm}_{2} \mathrm{Fe}_{16}$ compound under hydrostatic pressures up to $1 \mathrm{GPa}$ were studied. We have revealed that the high-temperature ferrimagnetic state easily magnetized in the basal plane is very sensitive to the volume changes and even moderate pressure is sufficient to its complete suppression and transformation to a helimagnetic state. At the same time, the lowtemperature ferrimagnetic state easily magnetized along the hexagonal axis does not disappear under pressure and the temperature of its transition to the high-temperature magnetic states increases under pressure. The remarkable stability of the ground ferrimagnetic state under external pressure can be attributed to the strengthening of the uniaxial magnetic anisotropy and to the mutual perpendicular orientation of the magnetic moments in the ground and the high-temperature magnetic states.
\end{abstract}

\section{Introduction}

The majority of $\mathrm{R}_{2} \mathrm{Fe}_{17}$ intermetallics $(\mathrm{R}=$ rare earth or $\mathrm{Y})$ exhibit a collinear ferro- or ferrimagnetic arrangement of magnetic moments depending on the presence of the light- or heavy-R element, respectively. The $\mathrm{R}_{2} \mathrm{Fe}_{17}$ intermetallics with small $\mathrm{R}$-ions are the exceptions; incommensurate helimagnetic structure has been described in the $\mathrm{Ce}_{2} \mathrm{Fe}_{17}, \mathrm{Lu}_{2} \mathrm{Fe}_{17}$ and $\mathrm{Tm}_{2} \mathrm{Fe}_{17}$ intermetallics below the Néel temperature $T_{\mathrm{N}}$ [1-3]. The spontaneous magnetization was observed at temperatures below a critical temperature $\Theta_{\mathrm{T}}<T_{\mathrm{N}}$. Between $\Theta_{\mathrm{T}}$ and $T_{\mathrm{N}}$, the magnetic moments of $\mathrm{Fe}$ ions are parallel to each other and antiparallel to those of $\mathrm{Tm}$ ions (in $\left.\mathrm{Tm}_{2} \mathrm{Fe}_{17}\right)$ in the atomic layers perpendicular to the crystalline $c$-axis, and they rotate by a certain angle in the consecutive layers. $\mathrm{Tm}_{2} \mathrm{Fe}_{17}$ is a collinear ferrimagnet below the transition temperature $\Theta_{\mathrm{T}}[1,4]$. Unlike $\mathrm{Ce}_{2} \mathrm{Fe}_{17}$ and $\mathrm{Lu}_{2} \mathrm{Fe}_{17}, \mathrm{Tm}_{2} \mathrm{Fe}_{17}$ is characterized by a spontaneous spinreorientation transition at $T_{\mathrm{sr}}$ due to the competition between the uniaxial anisotropy of the Tm-subsystem and the planar-type anisotropy of the Fe-subsystem [4]. Both the $\mathrm{Tm}$ and $\mathrm{Fe}$ magnetic moments in $\mathrm{Tm}_{2} \mathrm{Fe}_{17}$ orient perpendicular to the hexagonal $c$-axis between the magnetic ordering temperature $T_{\mathrm{N}}$ and $T_{\mathrm{sr}}$, and they are parallel to this axis below $T_{\text {sr. }}$.

The ferromagnetic state in $\mathrm{Ce}_{2} \mathrm{Fe}_{17}$ and $\mathrm{Lu}_{2} \mathrm{Fe}_{17}$ is rather unstable and transforms to a helimagnetic state under moderate hydrostatic pressure [2, 3]. This transformation is explained based on the hypothesis of a strong competition of the positive and negative $\mathrm{Fe}-\mathrm{Fe}$ exchange interactions due to the critical dependence of these interactions on the interatomic Fe-Fe distance [1]. In the present paper, variations of the temperatures of the magnetic phase transitions in the non-stoichiometric $\mathrm{Tm}_{2} \mathrm{Fe}_{16}$ compound [4] under a hydrostatic pressure of up to $1 \mathrm{GPa}$ is studied. $\mathrm{Tm}_{2} \mathrm{Fe}_{16}$ is a ferrimagnet [4] unlike $\mathrm{Tm}_{2} \mathrm{Fe}_{17}$ and non-stoichiometric $\mathrm{Tm}_{2} \mathrm{Fe}_{18}$ and $\mathrm{Tm}_{2} \mathrm{Fe}_{19}$ compounds having the same magnetic states [4]. The non-stoichiometric $\mathrm{Tm}_{2} \mathrm{Fe}_{16}-\mathrm{Tm}_{2} \mathrm{Fe}_{19}$ compounds crystallize in the disordered variant of the hexagonal $\mathrm{Th}_{2} \mathrm{Ni}_{17}$-type structure due to the existence of the new defected atomic positions as it was discussed in Ref. [4].

\section{Experimental details}

The $\mathrm{Tm}_{2} \mathrm{Fe}_{16}$ alloy was prepared by induction melting in Ref. [4]. The ingot was homogenized at $1450 \mathrm{~K}$ for $10 \mathrm{~h}$ and then quenched in water. The phase composition of the sample was investigated by microprobe analysis, $\mathrm{X}$ ray diffraction using $\mathrm{Cr}$ radiation, neutron diffraction and magnetometric analysis (see details in [4]). X-ray and neutron diffraction analysis indicated that the $\mathrm{Tm}_{2} \mathrm{Fe}_{16}$ compound crystallizes in the disordered variant of the hexagonal $\mathrm{Th}_{2} \mathrm{Ni}_{17}$-type structure [4]. The fraction of free $\alpha-\mathrm{Fe}$ was found to be not larger than $1 \mathrm{wt} . \%$. The magnetization measurements at a high hydrostatic pressure of up to $1 \mathrm{GPa}$ (at $5 \mathrm{~K}$ ) were performed in a SQUID magnetometer (Quantum Design Co.) using a miniature piston-cylinder $\mathrm{CuBe}$ pressure cell with a mixture of mineral oils as a pressure transmitting medium [5]. The pressure was determined at low temperatures using the known pressure dependence of the critical temperature of the superconducting state of the pure $\mathrm{Pb}(5 \mathrm{~N})$ sample. The high-pressure studies were performed in magnetic fields up to $7 \mathrm{~T}$ in the temperature range from 5 to $320 \mathrm{~K}$. A polycrystalline sample was used for the magnetic measurements. The evolutions of the transition temperature $\Theta_{\mathrm{T}}$, the Néel temperature $T_{\mathrm{N}}$ and the spin-reorientation temperature $T_{\text {sr }}$ under different pressures were determined from the

\footnotetext{
* Corresponding author: kuchin@imp.uran.ru
} 
temperature dependences of the magnetization $M(T)$ measured in a magnetic field of $0.01 \mathrm{~T}$ with the heating rate of $1 \mathrm{~K}$ per minute. $T_{\mathrm{C}}$ or $\Theta_{\mathrm{T}}$ were defined as a minimum of derivative of $M(T)$ or (at high pressures, see text) as a peak in $M(T)$ in the range $\sim(130-270) \mathrm{K} . T_{\mathrm{N}}$ was obtained as a local maximum in $M(T)$ in the range (230-250) K. $T_{\text {sr }}$ was defined as a peak in $M(T)$ in the range $\sim(90-130) \mathrm{K}$.

\section{Experimental results}

The temperature dependences of the magnetization $M(T)$ of $\mathrm{Tm}_{2} \mathrm{Fe}_{16}$ measured at $0.01 \mathrm{~T}, 0.1 \mathrm{~T}$ and $1 \mathrm{~T}$ under ambient and high pressures are presented in Fig. 1.

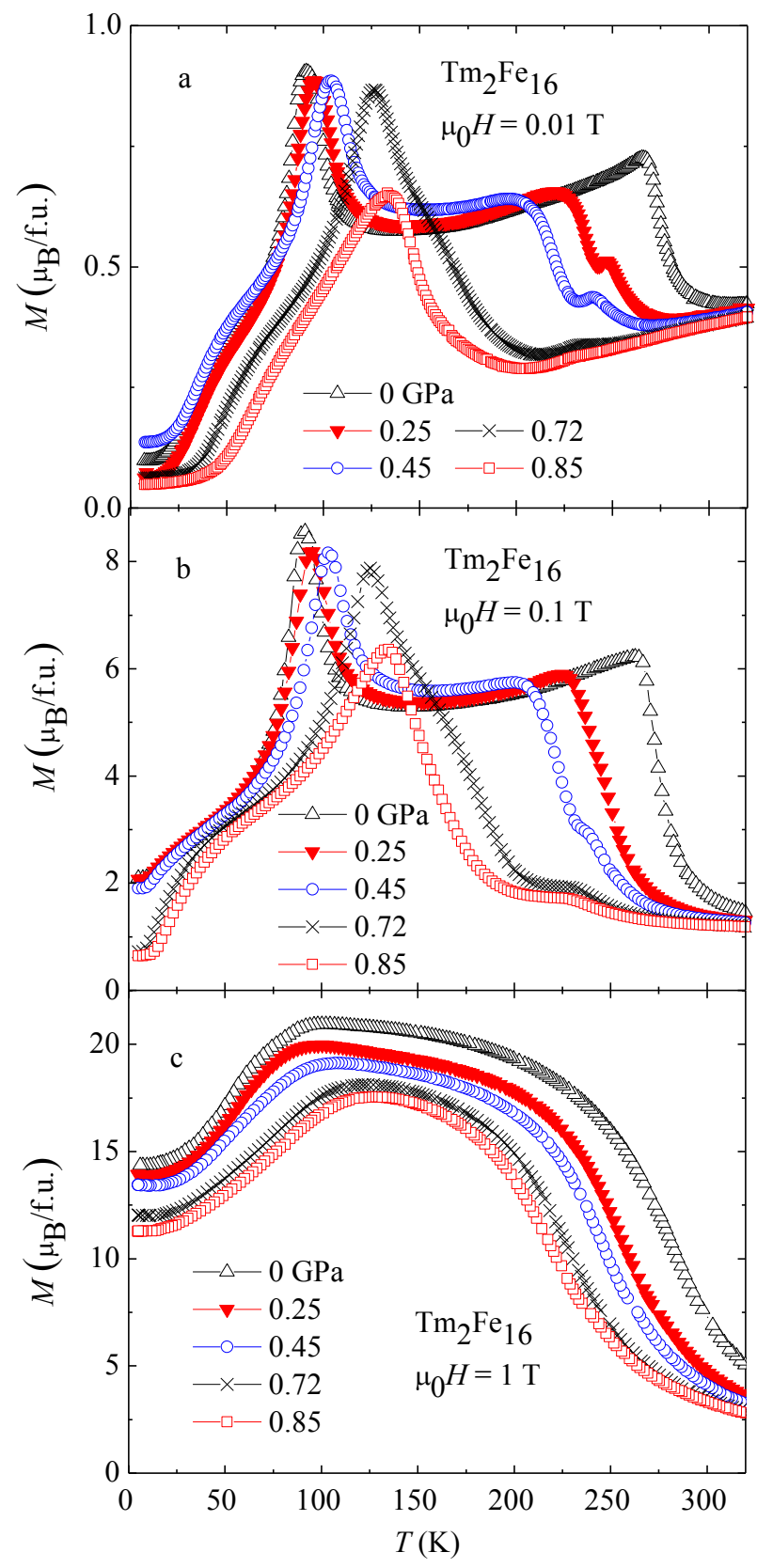

Fig. 1. Temperature dependences of magnetization of the $\mathrm{Tm}_{2} \mathrm{Fe}_{16}$ polycrystal measured in a field of $0.01 \mathrm{~T}(\mathrm{a}), 0.1 \mathrm{~T}(\mathrm{~b})$ and $1 \mathrm{~T}$ (c) under different pressures: $0,0.25,0.45,0.72,0.85$ $\mathrm{GPa}$ (right-to-left).
The drastic rise of $M(T)$ at $T \sim 90 \mathrm{~K}$ is a result of the well-known spontaneous spin-reorientation magnetic phase transition from the hexagonal axis to the basal plane. The large peak at $T \sim 90 \mathrm{~K}$ and the bump at $T<$ $T_{\text {sr }}$ in the $M(T)$ curves in Figs. 1a,b are the contributions from the magnetizations of the microcrystals whose $c$ axis is oriented along the external magnetic field [4].

$\mathrm{Tm}_{2} \mathrm{Fe}_{16}$ is a ferrimagnet under ambient pressure [4]. However, a small peak in the $M(T)$ curve at the Néel temperature is appeared under the pressure $0.25 \mathrm{GPa}$ in a field of $0.01 \mathrm{~T}$. Starting from this pressure, the types of magnetic states in $\mathrm{Tm}_{2} \mathrm{Fe}_{16}$ in a field of $0.01 \mathrm{~T}$ become the same as in $\mathrm{Tm}_{2} \mathrm{Fe}_{17}$ under ambient pressure (see Introduction). A field of 0.1 or $1 \mathrm{~T}$ destroys this induced helimagnetic state (Figs. 1b,c). The higher is the pressure, the stronger is the field to destroy the helimagnet. The increase in the magnetization under pressure at the temperatures below $T_{\mathrm{N}}$ is associated with the transition from the pressure-induced helimagnet to the ferrimagnetic state at $\Theta_{\mathrm{T}}$. In the range $P=0.25-0.72$ $\mathrm{GPa}, \Theta_{\mathrm{T}}$ characterizes the transition between the ferrimagnet and helimagnet, both easily magnetized in the basal plane. Under pressure, $T_{\mathrm{sr}}$ moves to the right, and $\Theta_{\mathrm{T}}$ and $T_{\mathrm{N}}$ move to the left along the $T$-axis of the plot in Fig. 1a. The lower is the temperature, the higher is the pressure to disappear the ferrimagnetic state easily magnetized in the basal plane as is followed from Fig. 1a. This regularity is supported by the magnetization curves measured at $T=150 \mathrm{~K}, 200 \mathrm{~K}$ or $250 \mathrm{~K}$ under $P$ $=0.85 \mathrm{GPa}, 0.72 \mathrm{GPa}$ or $0.25 \mathrm{GPa}$ and lower pressures in Figs. 2b,c,d. All of them have apparently different slopes of the initial magnetization portions. The ground ferrimagnetic state easily magnetized along the $c$-axis and the high-temperature helimagnetic state exist under all pressures applied. The existence of the lowtemperature spontaneous ferrimagnetism is supported by the same slope of the initial magnetization portions under all pressures applied at $T=50 \mathrm{~K}$ in Fig. 2a.

An intense peak in $M(T)$ is retained at $T \sim 135 \mathrm{~K}$ under $P=0.85 \mathrm{GPa}$ in Fig. 1a. It is obvious that this peak characterizes both the $T_{\mathrm{sr}}$ and $\Theta_{\mathrm{T}}$ values. $T_{\mathrm{sr}}$ grows as the pressure increases with the rate $\mathrm{d} T_{\mathrm{sr}} / \mathrm{d} P=49.4$ $\mathrm{K} / \mathrm{GPa}$. The pressure dependence of $\Theta_{\mathrm{T}}$ seems to be nonlinear, as is presented in Fig. 3. The linear fit of this dependence gives the average slope of $\mathrm{d} \Theta_{\mathrm{T}} / \mathrm{d} P=-122.6$ $\mathrm{K} / \mathrm{GPa}$ (Fig. 3). The pressure-induced Néel temperature decreases linearly as the pressure increases with a slope of $\mathrm{d} T_{\mathrm{N}} / \mathrm{d} P=-22.3 \mathrm{~K} / \mathrm{GPa}$, as is presented in Fig. 3 .

The samples are polycrystalline with a magnetic anisotropy field of about 6-7 $\mathrm{T}$ [4]. This is why we are not able to saturate the samples and, hence, it is not easy to determine the spontaneous magnetization. For example, $M(H)$ curves for $\mathrm{Tm}_{2} \mathrm{Fe}_{16}$ at $T=50-250 \mathrm{~K}$ under different pressures are presented in Fig. 2. Therefore, we estimate the values of the pressureinduced decrease in the magnetization from the values observed in a field of $5 \mathrm{~T}$. The results are presented in Fig. 4. The value $\mathrm{d}(\ln M) / \mathrm{d} P=-2 \cdot 10^{-3} \mathrm{GPa}^{-1}$ at $5 \mathrm{~K}$ for $\mathrm{Tm}_{2} \mathrm{Fe}_{16}$ is the quite a reasonable value for the $\mathrm{R}_{2} \mathrm{Fe}_{17}$ compounds, compared with $\mathrm{d}(\ln M) / \mathrm{d} P=-8.2 .10^{-4} \mathrm{GPa}^{-1}$ for $\mathrm{Y}_{2} \mathrm{Fe}_{17}$ in Ref. [6]. 


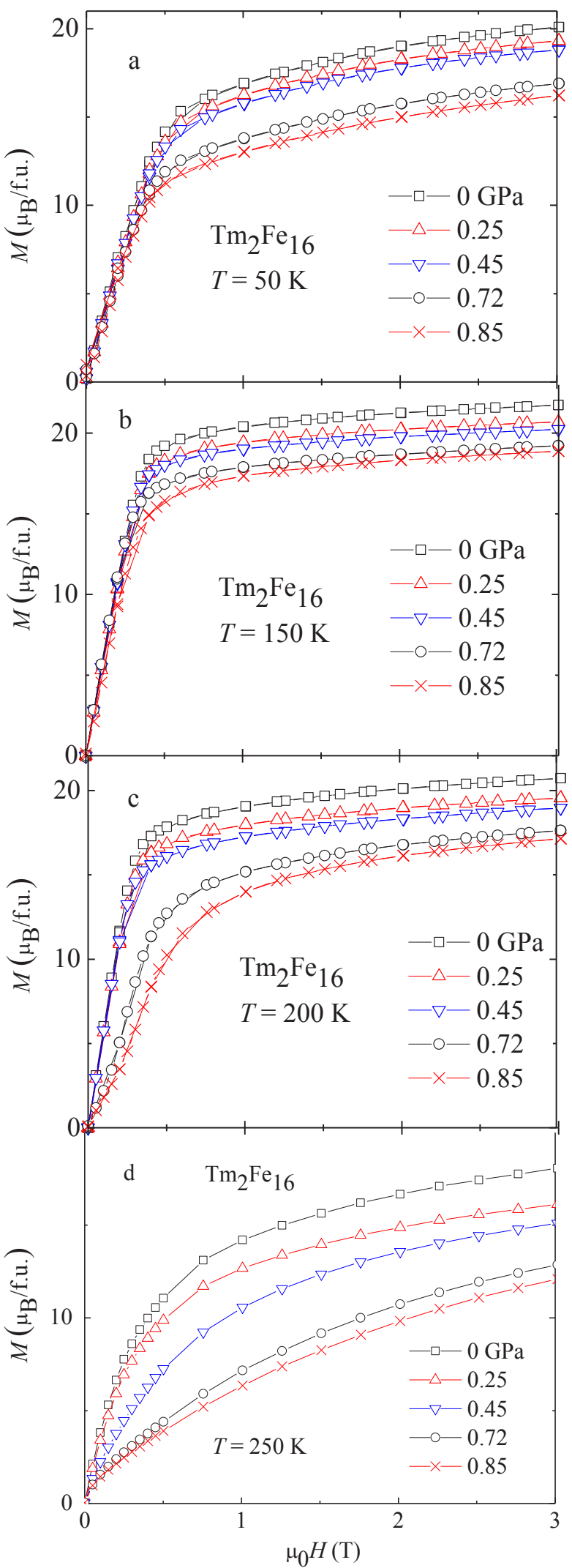

Fig. 2. Magnetic isotherms of $\mathrm{Tm}_{2} \mathrm{Fe}_{16}$ measured at $T=50 \mathrm{~K}$ (a), $150 \mathrm{~K}$ (b), $200 \mathrm{~K}$ (c) and $250 \mathrm{~K}$ under pressures: 0, 0.25, $0.45,0.72,0.85 \mathrm{GPa}$ (top-down).

The pressures on the dependences of magnetization versus temperature or pressure in Figs. 1, 2, 4 are the pressures at low temperatures. For the pressure dependences of the transition temperatures in Fig. 3 we use the corrected pressures at the temperatures of the transitions.

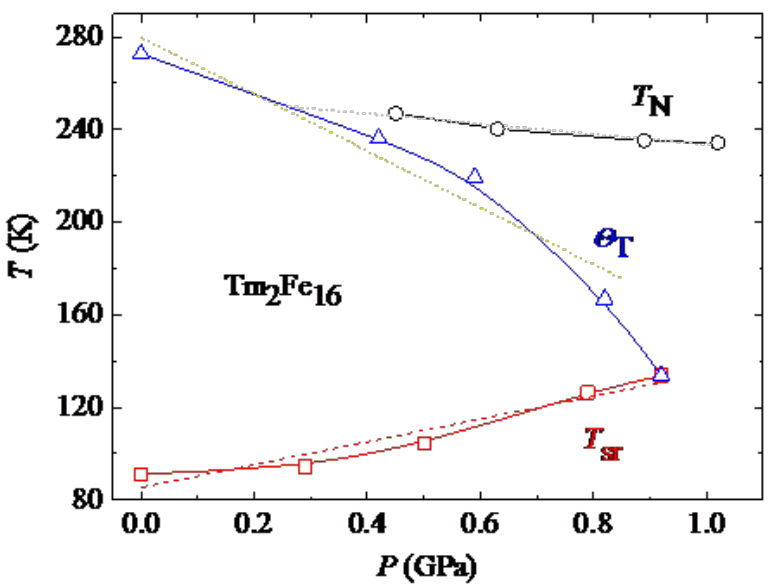

Fig. 3. Pressure dependences of $T_{\mathrm{N}}(\mathrm{o}), \Theta_{\mathrm{T}}(\Delta)$ and $T_{\mathrm{sr}}(\square)$ of $\mathrm{Tm}_{2} \mathrm{Fe}_{16}$ measured at $0.01 \mathrm{~T}$. $T_{\mathrm{C}}(\Delta)$ under $P=0 \mathrm{GPa}$. The dashed lines are the linear fits of the dependences.

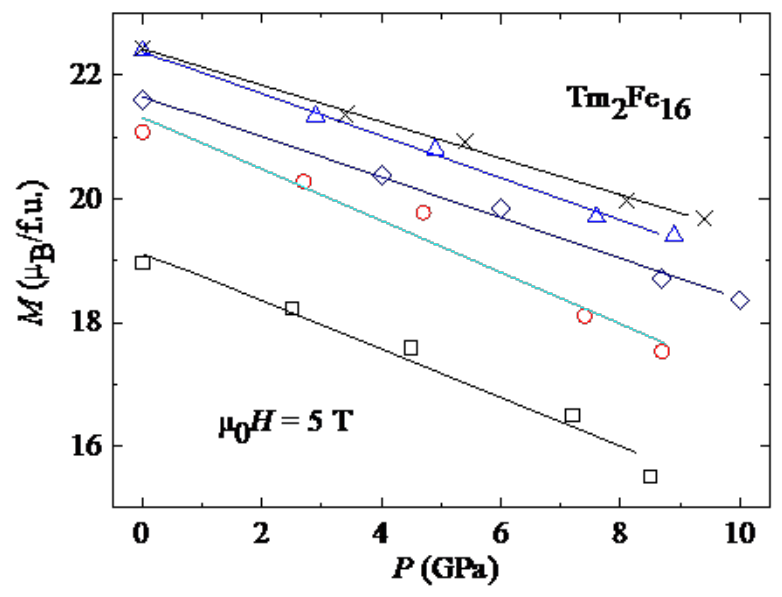

Fig. 4. Pressure dependences of the magnetization of $\mathrm{Tm}_{2} \mathrm{Fe}_{16}$ measured in a field of $5 \mathrm{~T}$ at $T=5 \mathrm{~K}(\square), 50 \mathrm{~K}(\mathrm{o}), 100 \mathrm{~K}(\Delta)$, $150 \mathrm{~K}(\times), 200 \mathrm{~K}(\diamond)$. The lines are the linear fits of the dependences.

\section{Discussion}

The increase in the negative exchange interactions in $\mathrm{Tm}_{2} \mathrm{Fe}_{16}$ under pressure (Figs. 1a,b) is analogous to the increase in the negative exchange interactions in the system $\mathrm{Tm}_{2} \mathrm{Fe}_{16}, \mathrm{Tm}_{2} \mathrm{Fe}_{17}, \mathrm{Tm}_{2} \mathrm{Fe}_{18}$ and $\mathrm{Tm}_{2} \mathrm{Fe}_{19}$, as the $\mathrm{Fe}$ content increases in Ref. [4]. The behavior of $\mathrm{Tm}_{2} \mathrm{Fe}_{16}$ under pressure is rather similar to the cases of $\mathrm{Ce}_{2} \mathrm{Fe}_{17}$ [2] and $\mathrm{Lu}_{2} \mathrm{Fe}_{17}$ [3]. This is because of the strong competition of the positive and negative Fe-Fe exchange interactions due to the critical dependence of these interactions on the interatomic Fe-Fe distance [1]. The similar values of the pressure dependences of the Néel temperature $\mathrm{d} T_{\mathrm{N}} / \mathrm{d} P=-22.3,-19$ and $-17 \mathrm{~K} / \mathrm{GPa}$ were obtained for $\mathrm{Tm}_{2} \mathrm{Fe}_{16}$ (Fig. 3), $\mathrm{Lu}_{2} \mathrm{Fe}_{17}$ and $\mathrm{Ce}_{2} \mathrm{Fe}_{17}$ [2, 3], 
respectively. The closeness of these values of $\mathrm{d} T_{\mathrm{N}} / \mathrm{d} P$ let us suggest that the high-temperature pressure-induced state in $\mathrm{Tm}_{2} \mathrm{Fe}_{16}$ is a helimagnet, as is in the other alloys under ambient pressure. The obtained pressure derivative of the temperature of the transition from the hightemperature ferrimagnet to the helimagnetic state $\mathrm{d} \Theta_{\mathrm{T}} / \mathrm{d} P$ for $\mathrm{Tm}_{2} \mathrm{Fe}_{16}(-122.6 \mathrm{~K} / \mathrm{GPa})$ is of the same order as for $\mathrm{Ce}_{2} \mathrm{Fe}_{17}(-380 \mathrm{~K} / \mathrm{GPa}[2])$ and $\mathrm{Lu}_{2} \mathrm{Fe}_{17}(-195 \mathrm{~K} / \mathrm{GPa}[3])$. They are also by one order higher than the pressure derivatives of the Néel temperature. Feasibly, this similar correlation between $\mathrm{d} T_{\mathrm{N}} / \mathrm{d} P$ and $\mathrm{d} \Theta_{\mathrm{T}} / \mathrm{d} P$ for $\mathrm{Tm}_{2} \mathrm{Fe}_{16}, \mathrm{Lu}_{2} \mathrm{Fe}_{17}$ and $\mathrm{Ce}_{2} \mathrm{Fe}_{17}$ can be explained by the affinity of the magnetic states realized in these compounds.

As a rule, a hydrostatic pressure leads to the decrease in the temperature of the ferro- or ferrimagnetic ordering in $\mathrm{R}_{2} \mathrm{Fe}_{17}[2,3,6]$. The low-temperature ferromagnetic phase in the $\mathrm{Lu}_{2} \mathrm{Fe}_{17}$ and $\mathrm{Ce}_{2} \mathrm{Fe}_{17}$ compounds disappears completely above 0.5 and $0.25 \mathrm{GPa}$, respectively [2, 3]. As for $\mathrm{Tm}_{2} \mathrm{Fe}_{16}$, the temperature $T_{\mathrm{sr}}$ of the transition from the ground ferrimagnetic state to the high-temperature magnetic states increases under pressure. Therefore, the low-temperature ferrimagnetic state is quite stable in this compound. The increase of $T_{\mathrm{sr}}$ in $\mathrm{Tm}_{2} \mathrm{Fe}_{16}$ under pressure is a result of the weakening of the planar-type magnetic anisotropy in the Fe-subsystem. The weakening of the easy-plane type magnetic anisotropy under pressure was obtained for $\mathrm{Y}_{2} \mathrm{Fe}_{17}$ in Ref. [6].

Two lines $\Theta_{\mathrm{T}}(P)$ and $T_{\mathrm{sr}}(P)$ in the magnetic phase diagram intersect each other at the critical point $(0.92$ GPa, 133.7 K) for $\mathrm{Tm}_{2} \mathrm{Fe}_{16}$ (Fig. 3). The ground collinear ferrimagnet easily magnetized along the hexagonal axis transforms entirely to the high-temperature helimagnet easily magnetized in the basal plane at this critical point.

\section{Conclusions}

The influence of the hydrostatic pressure on the $\Theta_{\mathrm{T}}, T_{\mathrm{N}}$ and $T_{\text {sr }}$ temperatures of the $\mathrm{Tm}_{2} \mathrm{Fe}_{16}$ compound has been studied: $\mathrm{d} \Theta_{\mathrm{T}} / \mathrm{d} P=-122.6 \mathrm{~K} / \mathrm{GPa}, \mathrm{d} T_{\mathrm{N}} / \mathrm{d} P=-22.3$, $\mathrm{d} T_{\mathrm{sr}} / \mathrm{d} P=49.4 \mathrm{~K} / \mathrm{GPa}$. The values of the pressure derivatives of $\Theta_{\mathrm{T}}$ and $T_{\mathrm{N}}$ for $\mathrm{Tm}_{2} \mathrm{Fe}_{16}$ are of the same order as for $\mathrm{Lu}_{2} \mathrm{Fe}_{17}$ and $\mathrm{Ce}_{2} \mathrm{Fe}_{17}$ [2, 3], apparently, due to the similar magnetic structures in these compounds [1]. It was revealed that the high-temperature ferrimagnetic state easily magnetized in the basal plane transforms entirely to the helimagnetic state under $P=$ $0.85 \mathrm{GPa}$ in $\mathrm{Tm}_{2} \mathrm{Fe}_{16}$. At the same time, the ground ferrimagnetic state easily magnetized along the hexagonal axis is retained under all pressures applied, and the temperature $T_{\mathrm{sr}}$ of its appearance increases under pressure. This remarkable stability of the ground ferrimagnetic state under external pressure can be attributed to the strengthening of the uniaxial magnetic anisotropy and to the mutual perpendicular orientation of the magnetic moments in the ground and hightemperature magnetic states. The magnetic phase diagram in the plane $(T-P)$ for the $\mathrm{Tm}_{2} \mathrm{Fe}_{16}$ compound is drawn. Two lines $\Theta_{\mathrm{T}}(P)$ and $T_{\mathrm{sr}}(P)$ in the magnetic phase diagram intersect each other at the critical point $(0.92$ GPa, 133.7 K).

\section{Acknowledgements}

The work was done within RAS Program "Magnet" (project № AAAA-A18-118020290129-5), with support of Grant Agency of CR (Project No. 15-03777S).

\section{References}

1. D. Givord, R. Lemaire, IEEE Trans. Magn. Mag-10, 109 (1974)

2. I.V. Medvedeva, Z. Arnold, A.G. Kuchin, J. Kamarád, J. Appl. Phys. 86, 6295 (1999)

3. J. Kamarád, Z.Arnold, I.V. Medvedeva, A.G. Kuchin, J. Magn. Magn. Mater. 242-245, 876 (2002)

4. A.G. Kuchin, S.P. Platonov, A.V. Korolyov, A.S. Volegov, V.I. Voronin, I.F. Berger, L.V. Elokhina, G.M. Makarova, E.V. Belozerov, J. Alloys Compd. 599, 26 (2014)

5. J. Kamarád, Z. Machatova, Z. Arnold, Rev. Sci. Instrum. 75, 5022 (2004)

6. J. Kamarád, O. Mikulina, Z. Arnold, B. GarciaLanda, M. R. Ibarra, J. Appl. Phys. 85, 4874 (1999) 